Supplement of Biogeosciences, 13, 223-238, 2016

http://www.biogeosciences.net/13/223/2016/

doi:10.5194/bg-13-223-2016-supplement

(C) Author(s) 2016. CC Attribution 3.0 License.

(c) (i)

Supplement of

\title{
The carbon cycle in Mexico: past, present and future of $\mathrm{C}$ stocks and fluxes
}

\section{G. Murray-Tortarolo et al.}

Correspondence to: G. Murray-Tortarolo (gnm202@exeter.ac.uk)

The copyright of individual parts of the supplement might differ from the CC-BY 3.0 licence. 


\section{Figure S1: Vegetation cover types}

All NLCT (9)

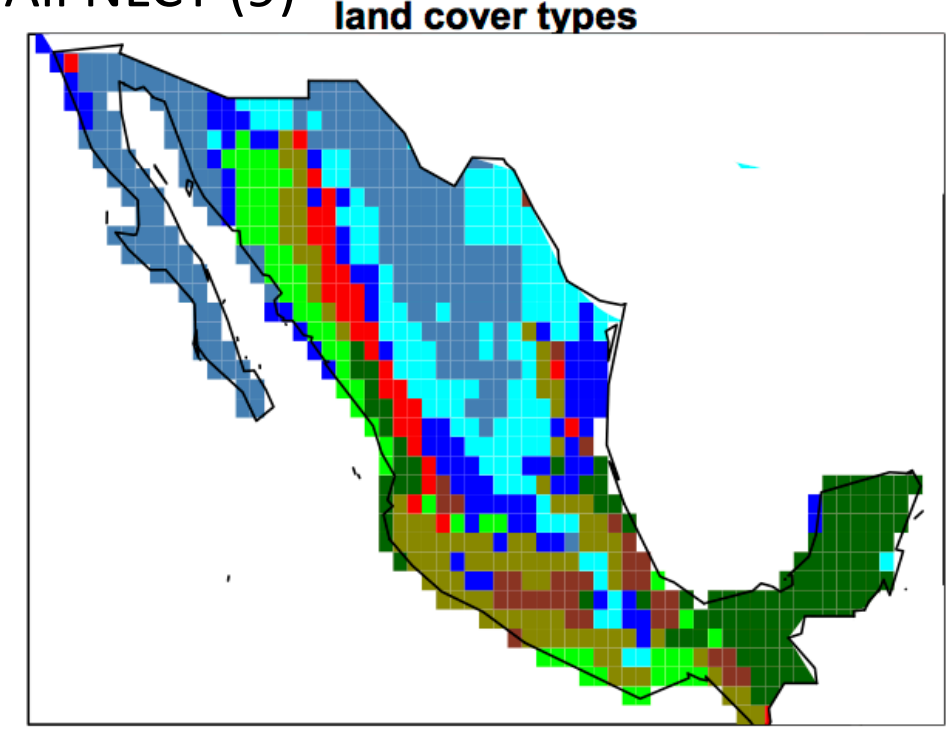

\section{LCT Natural Land Cover Types}

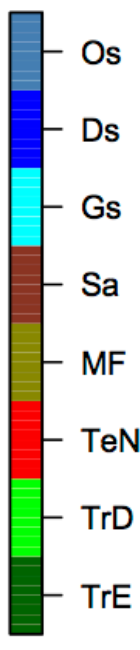

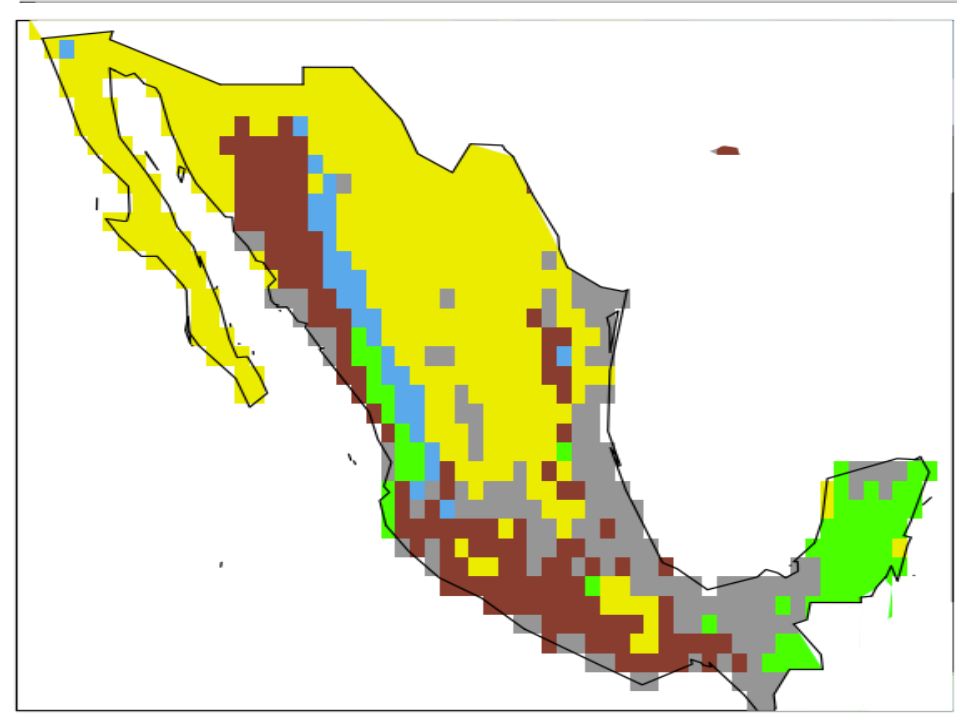

Supplementary 1: natural land cover types (right) and aggregated covers (left). OS: Open shrubland, Ds: Dense Shrubland; Gs: Grassland; Sa: Savanna; MF: Mixed Forest; TeN: Temperate Needleaf forest; TrD: Tropical Deciduous Forest, TrE: Tropical Evergreen forest.

We aggregate the natural land cover type (as derived directly from Ramankuty and Foley, 1999) into four functional types to simplify the analysis and masked the areas where the agricultural fraction what higher than $50 \%$. This four aggregated categories are in good agreement with the vegetation maps by Rzedowski (1978), except we aggregated all deciduous categories into one as they tend to co-exist at this scale. Additionally, we merged the shrublands and grasslands into one category for easier comparison against forested lands 
Figure S2. Sampling points for the field data (vegetation and soil)

Field Data Sampling Points

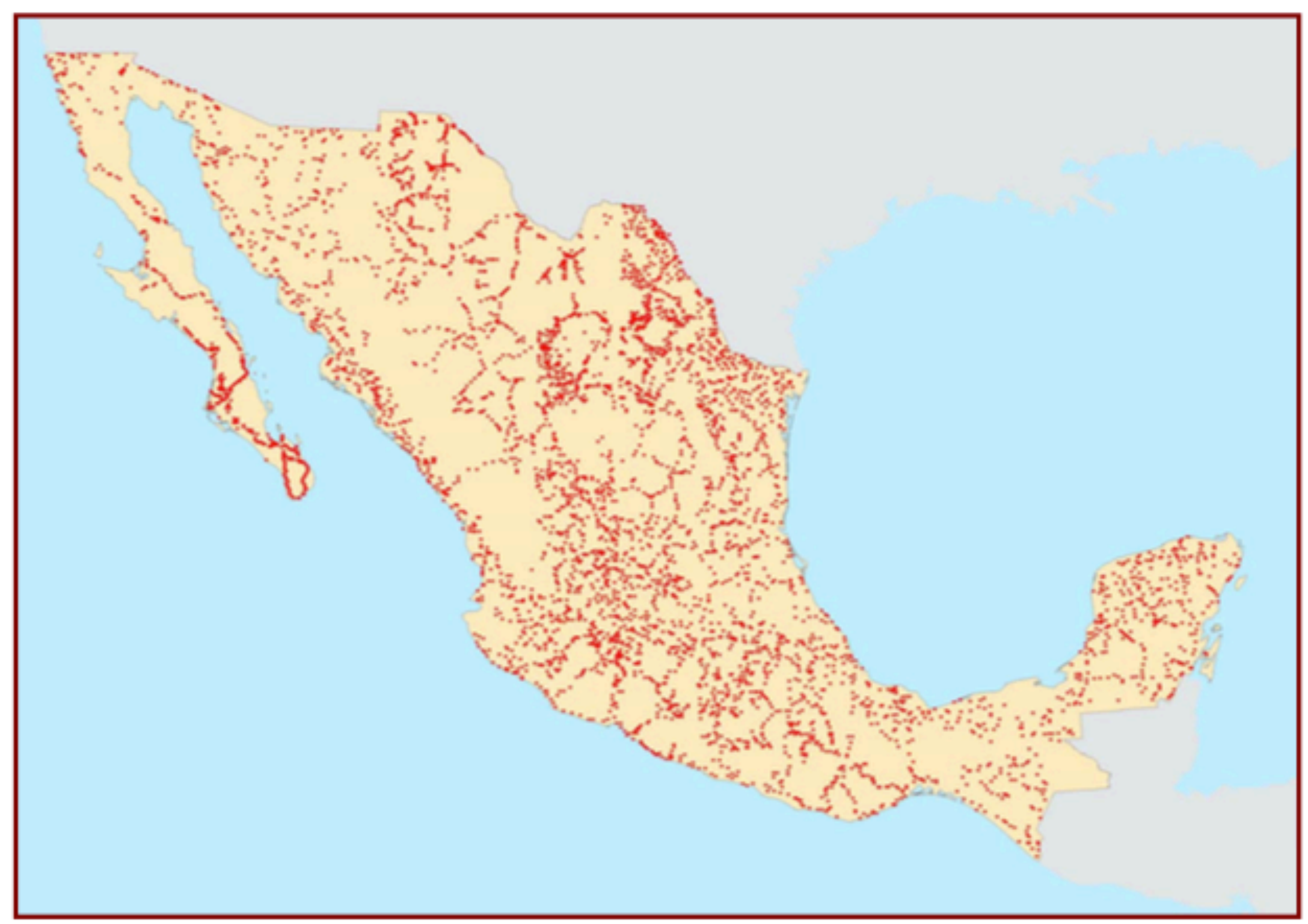


Figure S3: Gridded temperature trend for 2005-2100 for four RCPs. Stippled for $>66 \%$ model agreement.

Temperature change (2080:2099 - 1980:1999)
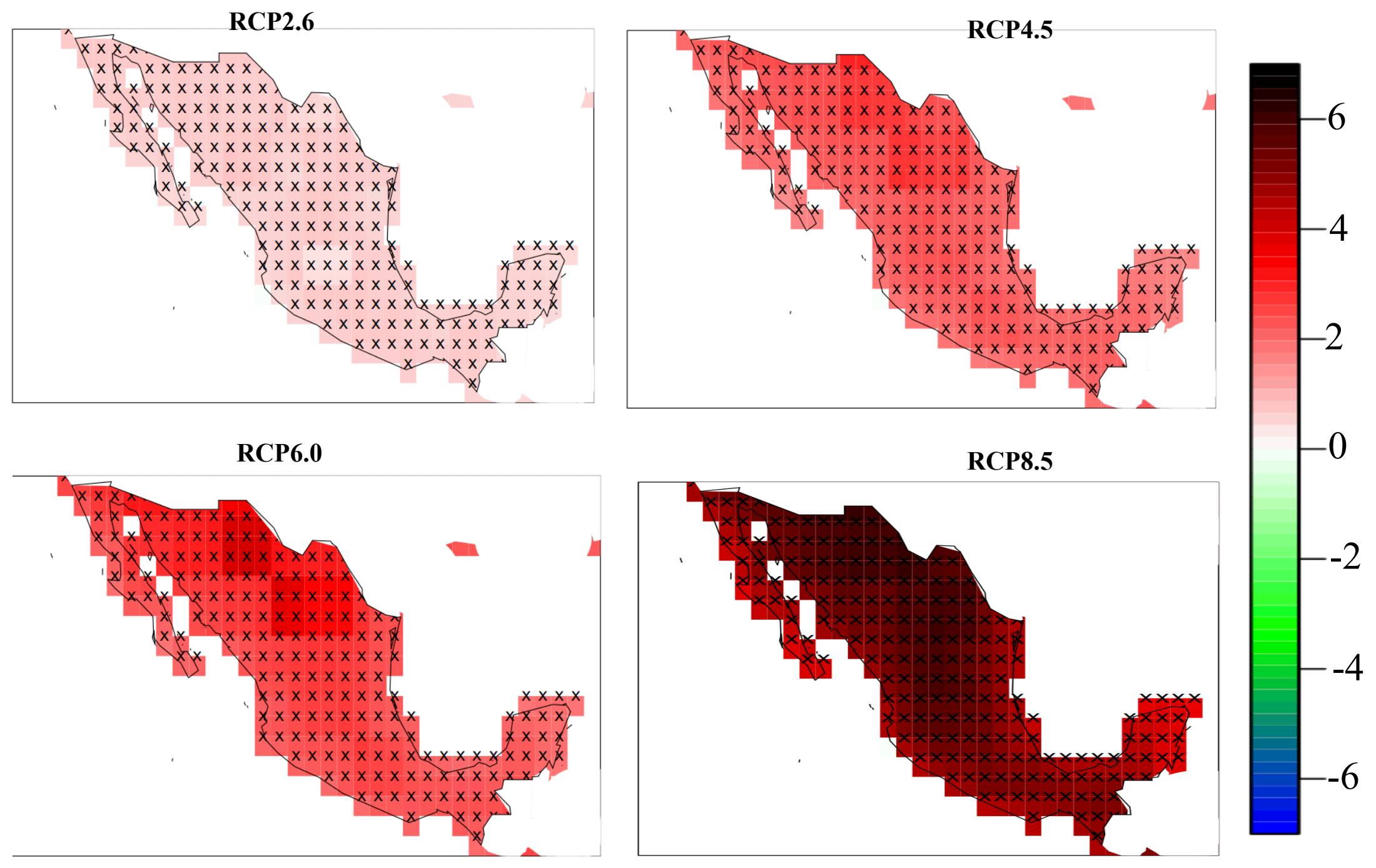

ڤ 
Figure S4: Gridded precipitation trend for $2005-2100$ for four RCPs. Stippled for $>66 \%$ model agre Precipitation change (2080:2099 - 1980:1999)

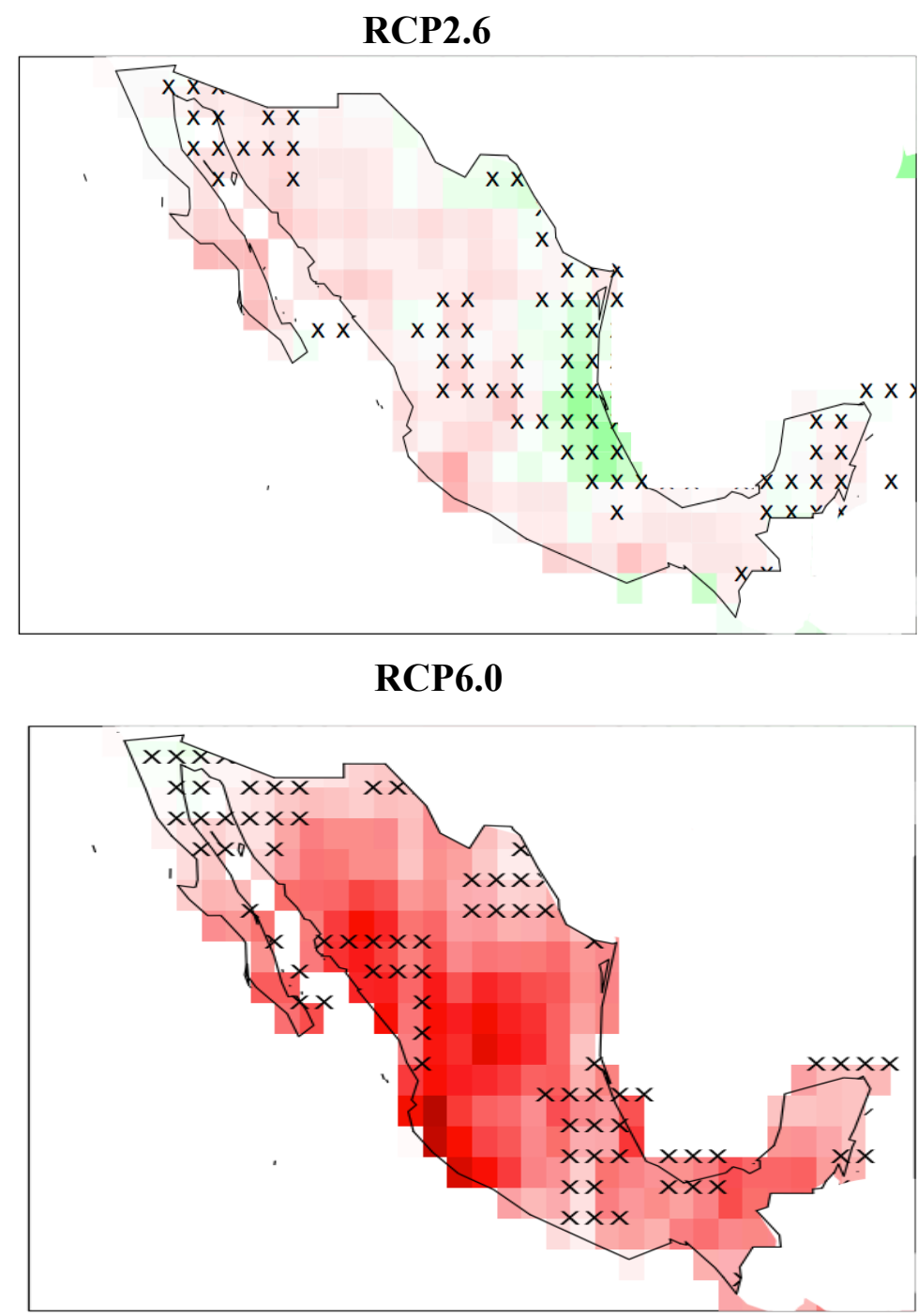

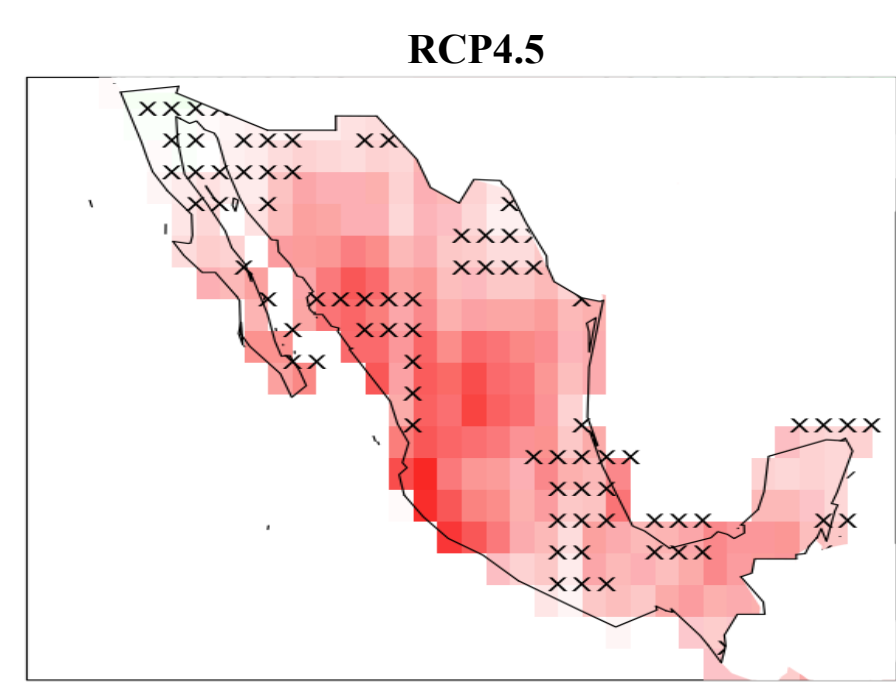

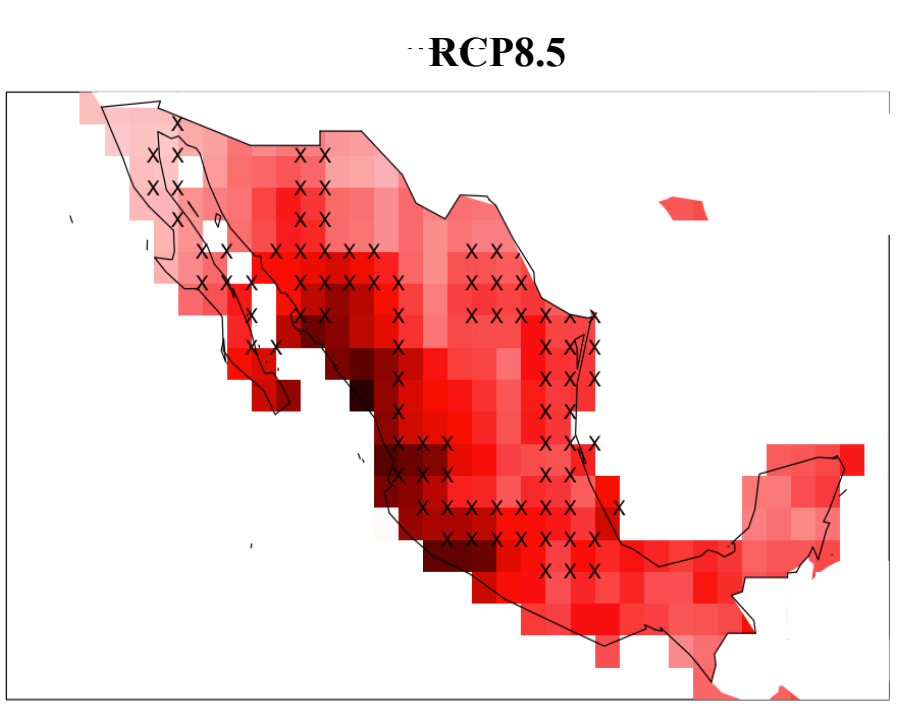

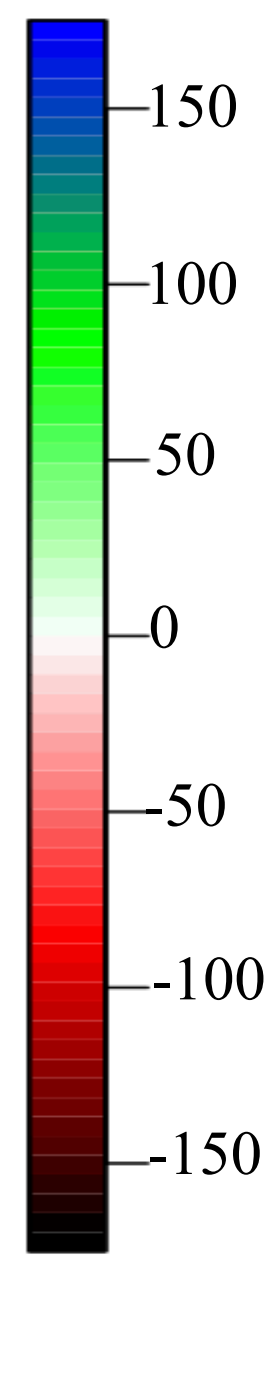

$\widehat{\Xi}$ 
Figure S5: Change in the mean residence time of C over the last 60 years (1950-2010).

\section{Carbon Mean Residence Time Trend (1901-2000)}

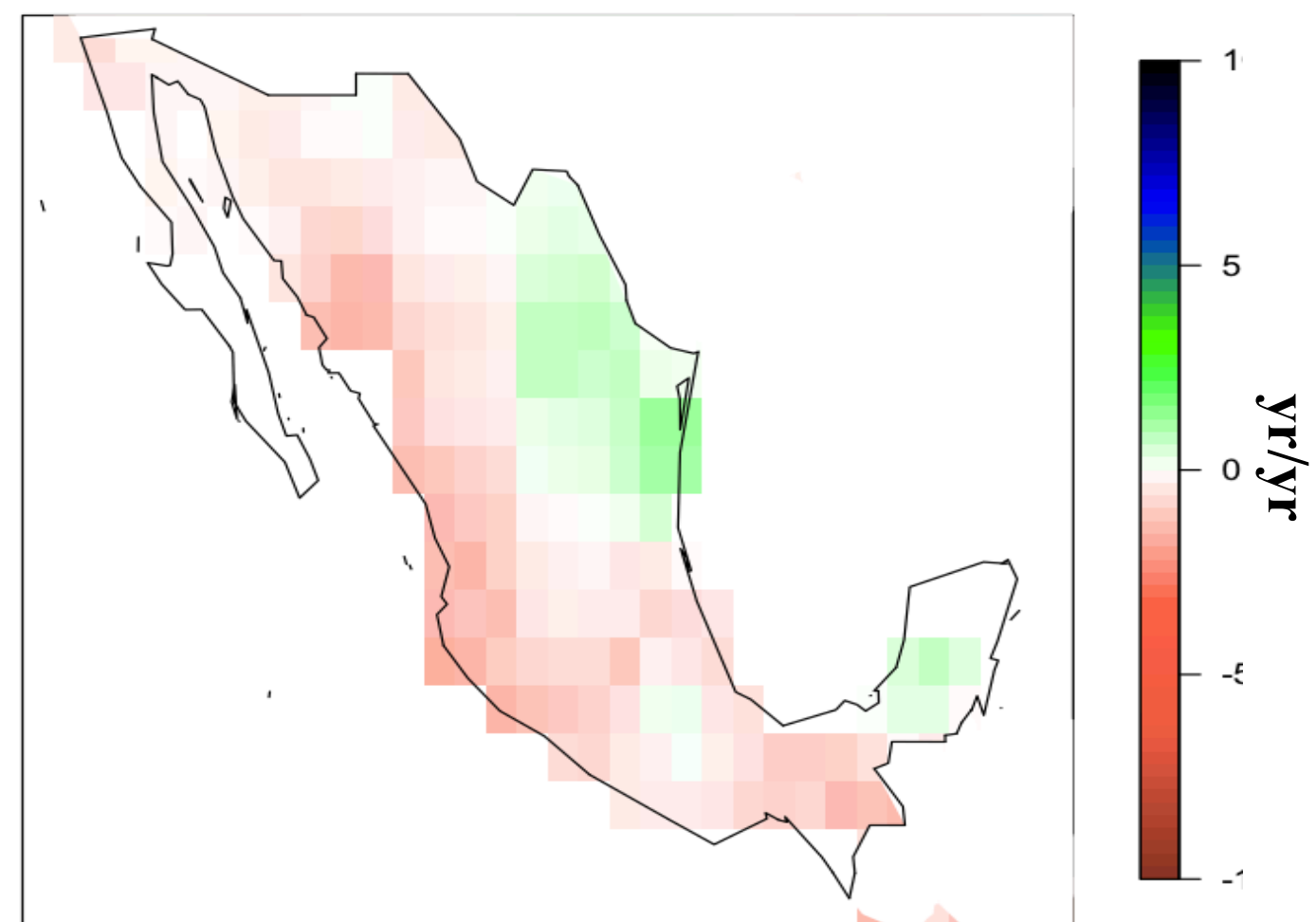

Supplementary 5: In the DGVMs soil C depends directly on vegetation input (i.e. litterfall) and accumulates slowly over time in different pools that are decomposed at different times (e.g. labile, intermediate and resistant). The soil microorganism use this $\mathrm{C}$ for their metabolism and as a consequence respire it as $\mathrm{CO} 2$ (Rh). Hence the actual content of soil $\mathrm{C}$ is a fine balance between vegetation inputs and $\mathrm{RH}$. Under stable conditions the two tend to be balance, but climate change alters this two fluxes. By dividing the soil $\mathrm{C}$ stock by the $\mathrm{Rh}$, we get a measurement of weather soil $\mathrm{C}$ is increasing or decreasing over time (i.e. weather we have a source or a sink of $\mathrm{C}$ in the soil).

For the particular case of Mexico, $\mathrm{Rh}$ is mostly negative, which means soil $\mathrm{C}$ is been lost faster that it is been accumulated. This is particularly important in future scenarios as temperature increases, because it can rapidly alter the country from been a sink of $\mathrm{C}$ to been a source. However, considerable uncertainties remain in our understanding of MRT and more direct observations are needed. 
Figure S6: Mean Land-Use-Change Emissions $\left(\mathrm{gC} \mathrm{m}^{-2} \mathrm{yr}^{-1}\right)$

Mean Land-Use-Change Emissions ( $\left(\mathrm{gC} \mathrm{m}^{-2} \mathrm{yr}^{-1}\right)$

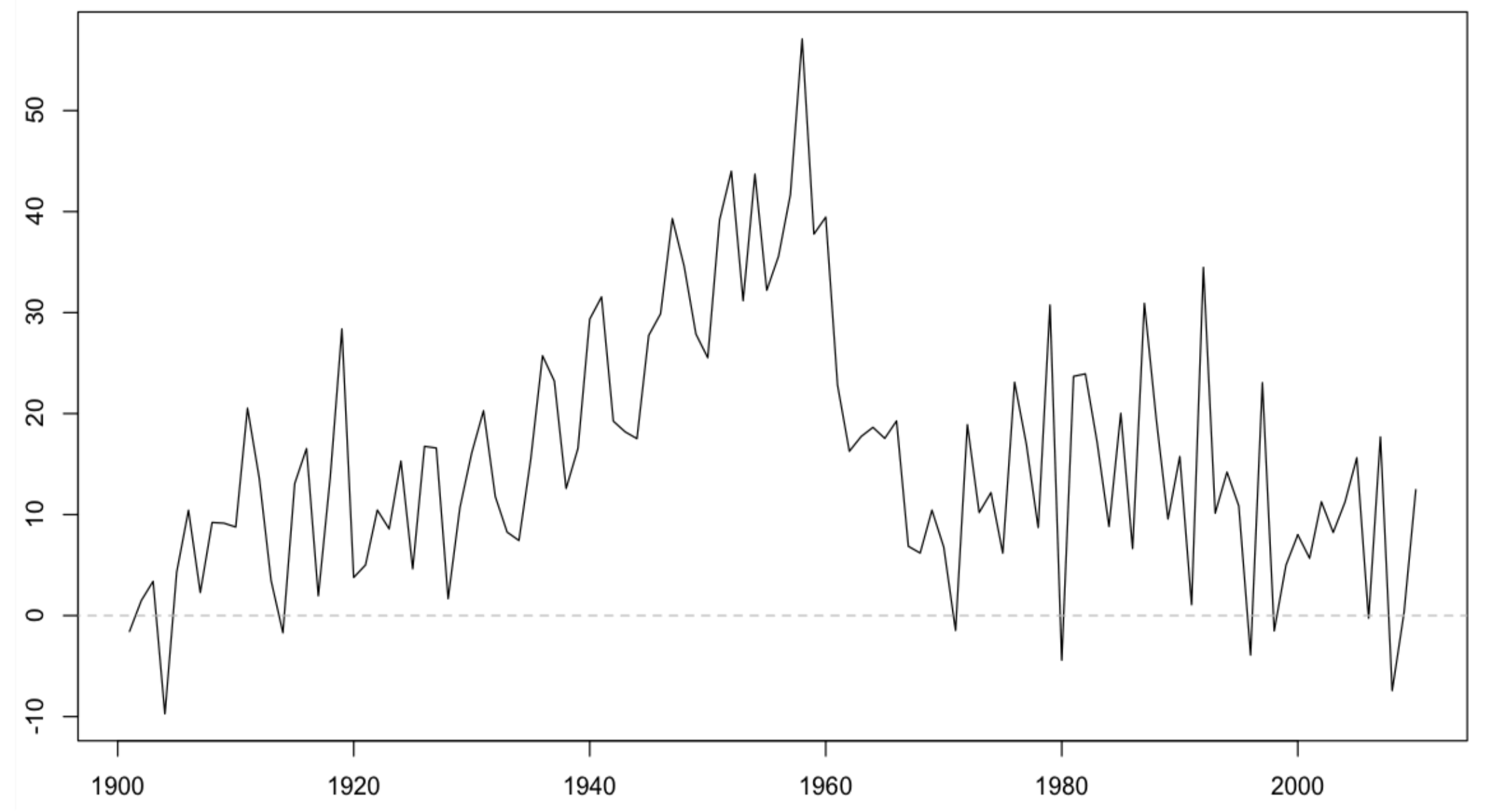


Figure S7: Modeled soil and vegetation C for the period 1990-2009

Vegetation C

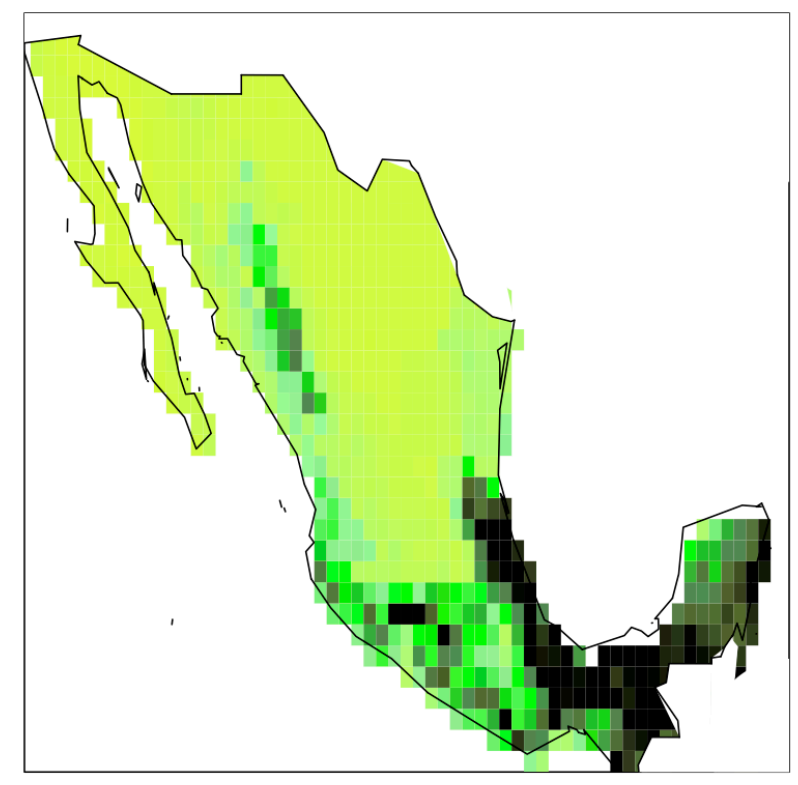

Soil C

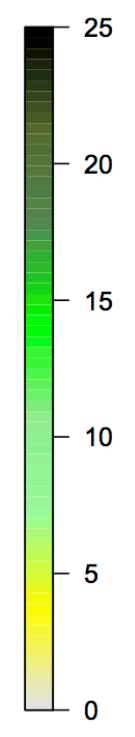

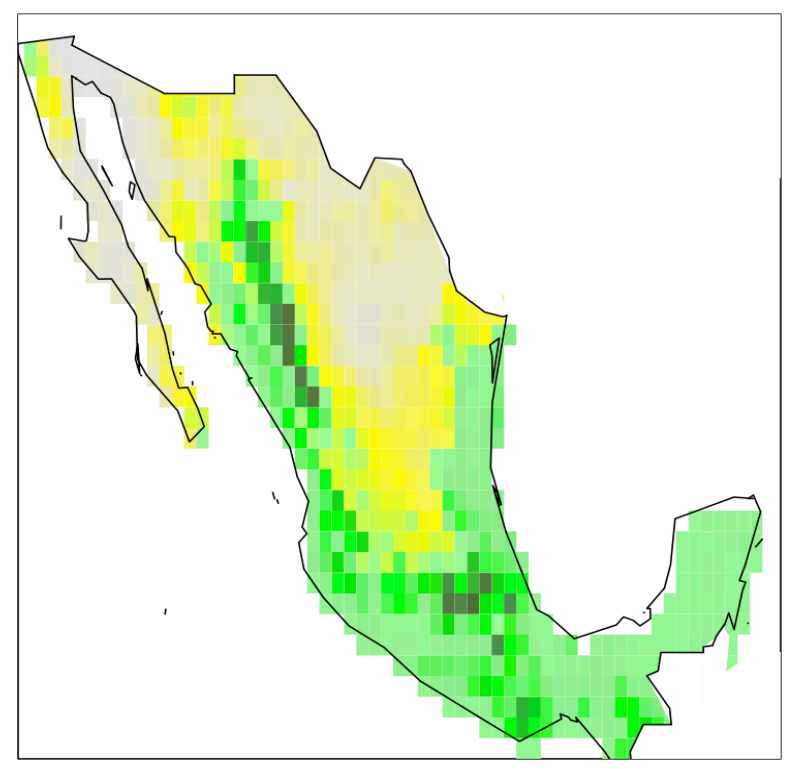

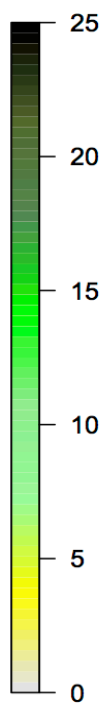

Supplementary 7: We calculated the Vegetation and Soil C in the DGVMs for an extended period of time (1990-2009) for the analysis on the present. We found that the country estimates for the period 2000-2005 (VegC: 20.3, SoilC 14.1 PgC) were similar than those for the longer time period (VegC: 20.2, SoilC 14.0 PgC). 
Table S1. Models used for the analysis.

\begin{tabular}{|l|l|}
\hline DGVMs & ESMs \\
\hline CLM4.5 & CCSM4 \\
\hline ISAM & GFDL-ESM2G \\
\hline LPJ & HadGEM2-ES \\
\hline LPJ-GUESS & IPSL-CM5A-LR \\
\hline LPX-Bern & IPSL-CM5A-MR \\
\hline JULES3.2 & MIROC-ESM \\
\hline OCN & MIROC-ESM-CHEM \\
\hline VEGAS & NorESM1-M \\
\hline VISIT & NorESM1-ME \\
\hline
\end{tabular}




\begin{tabular}{|l|c|c|c|c|c|c|}
\hline \multicolumn{1}{|c|}{ GPP } & \multicolumn{2}{c|}{ DGVM } & \multicolumn{2}{c|}{ Satellite } & \multicolumn{2}{c|}{ Fluxtower } \\
\hline Potential Land Cover Type & $\begin{array}{c}\text { Mean } \\
\left(\mathbf{g C ~}^{-2} \mathbf{y r} \mathbf{~}^{-1}\right)\end{array}$ & $\begin{array}{c}\text { Sum } \\
(\mathbf{T g C})\end{array}$ & $\begin{array}{c}\text { Mean } \\
\left(\mathbf{g C ~ m}^{-2} \mathbf{~ r ~ r}^{-1}\right)\end{array}$ & $\begin{array}{c}\text { Sum } \\
(\mathbf{T g C})\end{array}$ & $\begin{array}{c}\text { Mean } \\
\left(\mathbf{g C ~ m}^{-2} \mathbf{y r}^{-1}\right)\end{array}$ & $\begin{array}{c}\text { Sum } \\
(\mathbf{T g C})\end{array}$ \\
\hline Broadleaf evergreen forest & 2.16 & 555 & 2.29 & 588 & 2.01 & 516 \\
\hline Broadleaf deciduous forest & 1.11 & 486 & 1.25 & 547 & 1.20 & 525 \\
\hline Needleleaf evergreen forest & 1.41 & 129 & 1.42 & 130 & 1.57 & 144 \\
\hline Grassland/Shrubland & 0.62 & 463 & 0.58 & 433 & 0.49 & 366 \\
\hline Croplands & 1.15 & 486 & 1.19 & 503 & 1.27 & 537 \\
\hline National total & & 2119 & & 2203 & & 2089 \\
\hline
\end{tabular}


Table S3: Vegetation and Soil C for each product by NLCT

\begin{tabular}{|c|c|c|c|c|c|c|}
\hline Vegetation Carbon & & & & ITE & & \\
\hline Land Cover Type & $\begin{array}{c}\text { Mean } \\
\left({\left.\mathrm{gC} \mathrm{m}^{-2}\right)}\right.\end{array}$ & $\begin{array}{l}\text { Sum } \\
\text { (TgC) }\end{array}$ & $\begin{array}{c}\text { Mean } \\
\left({\left.\mathrm{gC} \mathrm{m}^{-2}\right)}\right.\end{array}$ & $\begin{array}{l}\text { Sum } \\
(\operatorname{TgC})\end{array}$ & $\begin{array}{c}\text { Mean } \\
\left(\mathrm{gC} \mathrm{m}^{-2}\right)\end{array}$ & $\begin{array}{l}\text { Sum } \\
(\operatorname{TgC})\end{array}$ \\
\hline Broadleaf evergreen forest & 23.8 & 6116 & 22.8 & 5859 & 22.1 & 5679 \\
\hline Broadleaf deciduous forest & 12.5 & 5475 & 11.9 & 5212 & 12.8 & 5606 \\
\hline Needleleaf evergreen forest & 15.2 & 1398 & 14.2 & 1306 & 15.8 & 1453 \\
\hline Grassland/Shrubland & 6.6 & 4930 & 6.3 & 4706 & 5.1 & 3809 \\
\hline Cropland & 7.4 & 3130 & 7.2 & 3045 & 7.8 & 3299 \\
\hline TOTALS & & 21050 & & 20129 & & 19864 \\
\hline
\end{tabular}

\begin{tabular}{|c|c|c|c|c|c|c|}
\hline \multirow{2}{*}{$\begin{array}{l}\text { Soil Carbon } \\
\text { Land Cover Type } \\
\end{array}$} & \multicolumn{2}{|c|}{ DGVMs } & \multicolumn{2}{|c|}{ FAO } & \multicolumn{2}{|c|}{ Field } \\
\hline & $\begin{array}{c}\text { Mean } \\
\left(\mathrm{gC}^{-2}\right)\end{array}$ & $\begin{array}{l}\text { Sum } \\
\text { (TgC) }\end{array}$ & $\begin{array}{c}\text { Mean } \\
\left(\mathrm{gC}^{-2}\right)\end{array}$ & $\begin{array}{l}\text { Sum } \\
\text { (TgC) }\end{array}$ & $\begin{array}{c}\text { Mean } \\
\left(\mathrm{gC}^{-2}\right)\end{array}$ & $\begin{array}{l}\text { Sum } \\
\text { (TgC) }\end{array}$ \\
\hline Broadleaf evergreen forest & 11.8 & 3032 & 12.5 & 3212 & 11.9 & 3058 \\
\hline Broadleaf deciduous forest & 8.9 & 3898 & 8.3 & 3625 & 9.4 & 4117 \\
\hline Needleleaf evergreen forest & 10.5 & 966 & 11.2 & 1030 & 11.0 & 2012 \\
\hline \multirow[t]{2}{*}{ Grassland/Shrubland } & 4.2 & 3137 & 4.5 & 3361 & 5.5 & 4108 \\
\hline & 5.8 & 2454 & 6.2 & 2622 & 6.7 & 2834 \\
\hline TOTALS & & 13487 & & 13862 & & 15130 \\
\hline
\end{tabular}




\section{Table S4: Links to freely available datasets used in this paper}

- Climate: http://www.cru.uea.ac.uk/high-resolution-gridded-datasets

- Land Cover: https://nelson.wisc.edu/sage/data-and-models/global-potential-vegetation/index.php

- Land Use Change: http://themasites.pbl.nl/tridion/en/themasites/hyde/

- DGVMS (Data used is version 2, not freely available, but version 1 is): http://www-Iscedods.cea.fr/invsat/RECCAP/V2/

- MTE (Fluxtowers):

https://climatedataguide.ucar.edu/climate-data/fluxnet-mte-multi-tree-ensemble

- ESMs (CMIP5): http://cmip-pcmdi.llnl.gov/cmip5/data portal.html

- Biomass (National Forest Inventory):

http://www.alianza-mredd.org/componentes/monitoreo-reporte-y-verificacion/productos/mapade-la-densidad-de-carbono-en-biomasa-lenosa-aerea-de-los-bosques-y-selvas-enmexico-2\#.VldkgGAlJst

- $\quad$ Soil C from FAO:

http://www.fao.org/soils-portal/soil-survey/soil-maps-and-databases/harmonized-world-soildatabase-v12/en/

- The rest of the data is available under request by contacting Guillermo Murray-Tortarolo (gnm202@exeter.ac.uk or gmurrayt@gmail.com) 